\author{
Military Technical College \\ Kobry El-Kobbah, \\ Cairo, Egypt.
}

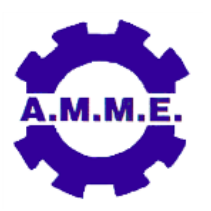

$17^{\text {th }}$ International Conference on Applied Mechanics and Mechanical Engineering.

\title{
DESIGN AND IMPLEMENTATION OF NAVIGATION CONTROL SYSTEM FOR UNMANNED GROUND VEHICLES
}

\author{
A. Khalil", S. Hegazy", A.Roheim* and Y. H. Hossam El-Din ${ }^{\ddagger}$
}

\begin{abstract}
This paper presents the design and implementation of a navigation control system for Unmanned Ground Vehicles (UGV). Three actuators for steering system, speed control and braking systems are designed and implemented to convert an electric car to be unmanned vehicle. A mathematical model for Ackerman steering is modeled and simulated using MATLAB/SIMULINK. The concept of structure of the system and the modularity and redundancy of the implemented control algorithms are emphasized during system development. The proposed navigation system includes longitudinal control and lateral control. Longitudinal control is responsible for controlling the speed of the vehicle through the accelerator actuator. Lateral control is responsible for controlling the direction by using steering motor. The proposed navigation control system is implemented based on GPS with an integrated digital compass. The distance error of the vehicle position is calculated according to vehicle heading. Of coarse the simulation path from simulation results, is coincident on the required path. Form experimental testing results there are good correlations for actual and required navigation between two points, three points and four points. There is a little difference between actual vehicle path and required path. This is due to using commercial (low-cost) sensors and actuators. The significance of this work is in the understanding of the transition from theory to practice and to integrate the necessary technology for local manufacturing of these types of vehicles.
\end{abstract}

\section{KEY WORDS}

Electric Vehicle, Unmanned Ground Vehicle (UGV), bicycle model and GPS, Navigation Control.

\footnotetext{
* Egyptian Armed Forces.

$\ddagger \quad$ Future University in Egypt (FUE).
} 


\section{NOMONCLATURE}

$\begin{array}{ll}d & \text { Rear wheel track of the vehicle } \\ k_{p}, k_{I}, k_{d} & \text { PID controller Gain parameters (proportional, integral and derivative) } \\ L & \text { Wheel base of the vehicle } \\ R & \text { Instantaneous radius of rotation } \\ T & \text { Motor or braking torque } \\ V_{s} & \text { Forward vehicle velocity } \\ V & \text { Velocity of the rear axle center point } \\ V_{x} & \text { Vehicle longitudinal speed } \\ \alpha & \text { Wheel angle rotation } \\ \omega & \text { Angular velocity } \\ \varphi & \text { Vehicle Heading angle (angle between vehicle } \mathrm{X} \text { axis and north) }\end{array}$

\section{INTRODUCTION}

Unmanned vehicles are devices that can move around and perform tasks without an operator onboard. It is normal for the operator of an unmanned vehicle to direct the behavior of the vehicle from a remote location. Unmanned vehicles are used in many applications such as mine detection, mine disposal, working in high radiation environments, surveillance and etc.[1]. According to robotic point of view, unmanned vehicles can be classified to Tele-operated and autonomous. The teleported vehicle system receives navigational guidance remotely from off board driver; while the autonomous vehicle can find its way (navigate) depending on sensors installed on board and processing resources. X. Liu and B. Dai [2], deducted that the Unmanned Ground Vehicles UGV will be the main weapon of armies in $21^{\text {st }}$ century. That deduction was the result of reviewing the history of the UGV in military field and studying the key technologies of autonomous systems in some latest examples of UGVs. H. Lim et al [3] presented a nonlinear model predictive tracking control strategy for a six-wheeled nonholonomic UGV. The tracking error was minimized using optimal control. The performance of proposed control was verified experimentally by six-wheeled mobile robot platform. M. Noor et al [4] developed a remote-operated multi-directional UGV. PIC microcontroller and Xbee Pro as transceivers were used to make a UGV prototype to verify the moving ability of the proposed UGV via actual practice. T. Czapla and J. Wrona [5] presented the history, current state of knowledge and future perspectives of unmanned technology application for military ground vehicles. In addition to, a detailed description of the development of unmanned technology. G. Wilson, et al [6] designed three prediction models for calculating maximum allowable velocity of UGV for traveling on rough unknown terrain. J. Lee, [7] designed a remote control station and its software architecture for efficient control of UGV in combat fields. The significant role of the UGVs in the future military operations was the motivation for that work [8]. M. YounGeun et al. [9], designed a speed control and path following algorithm which proposed test driving robot. The algorithms were verified and tested by computer simulation and T-car test. From analysis the results, the proposed speed control 
algorithm decreases the overshoot caused by nonlinear parts of the vehicle dynamics and the performance of the path following algorithm.

This paper presents the steps for converting an electric vehicle to be unmanned ground vehicle using three actuators for controlling steering, speed and braking systems. A mathematical model for the developed unmanned vehicle is simulated for path between two points and multi points using MATLAB/SIMULINK. For navigation, a driving algorithm is proposed using GPS and compass, and steering/speed/brake management. The proposed UGV is considered as a basic platform for researchers to test and integrate their efforts to produce full autonomous vehicle.

\section{PROPOSED UNMANNED VEHICLE}

An electric vehicle shown in Fig. 1 driven by 48-V DC electric motor installed on the rear axle is converted to unmanned vehicle UGV. Three actuators for steering, accelerating and braking systems are designed. First, a DC motor was integrated with a variable resistance (multi turn potentiometer) to work as a servo motor for controlling the steering of the vehicle (steered by wire). Second a linear actuator is installed to control the car speed. Third a linear actuator is installed to stop and brake the car. Table 1 lists detailed dimensions and specifications of the UGV.

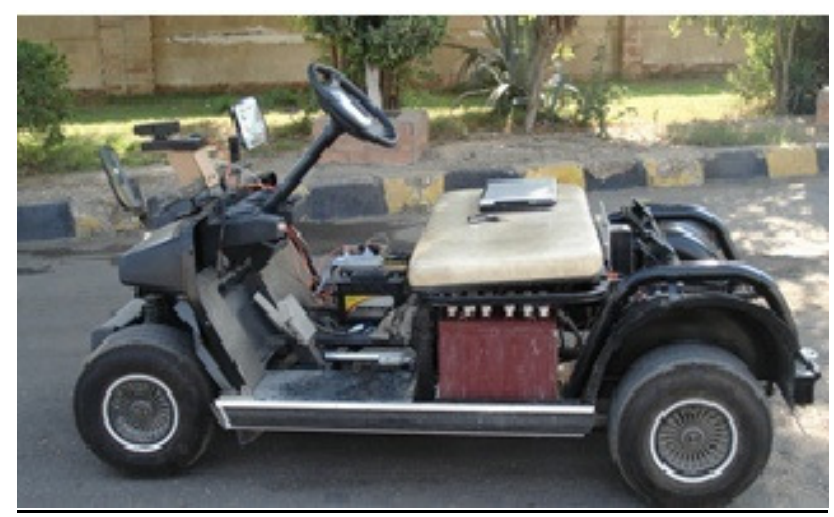

Fig. 1. Electric car platform.

Table 1. UGV specification.

\begin{tabular}{|c|c|c|}
\hline \multirow{3}{*}{ Dimension } & Length & $1.65 \mathrm{~m}$ \\
\cline { 2 - 3 } & Width & $1 \mathrm{~m}$ \\
\cline { 2 - 3 } & Height & $0.8 \mathrm{~m}$ \\
\hline \multicolumn{2}{|c|}{ Weight } & $600 \mathrm{~kg}$ \\
\hline \multicolumn{2}{|c|}{ Wheel Radius } & $0.15 \mathrm{~m}$ \\
\hline Max Speed & $0.5 \mathrm{~m} / \mathrm{s}$ \\
\hline \multicolumn{2}{|c|}{ Steering Type } & Ackerman Steering \\
\hline Driving Motor & $48 \mathrm{~V}$ \\
\hline \multirow{3}{*}{ Actuators } & steering & Servo motor 24 V \\
\cline { 2 - 3 } & Accelerator & Linear actuator 24 V \\
\cline { 2 - 3 } & brakes & Linear actuator 24 V \\
\hline \multirow{2}{*}{ Power } & Battery & 24 V NiCad Rechargeable $\times 2(1400 \mathrm{mAh})$ \\
\cline { 2 - 3 } & Operation Time & 2 h (Continuous Running) \\
\hline
\end{tabular}




\section{Actuators}

\section{(1) Steering system}

The steering system is controlled by $12 \mathrm{~V} \mathrm{DC}$ electric motor combined with chain sprocket mechanism installed on the steering column without removing the steering wheel to keep the option of regular driving. A variable resistance (multi turn potentiometer) is used to control the DC motor to work as a servo motor, shown in Fig. 2. A calibration of the steering system using electric motor and multi turn potentiometer is carried out.

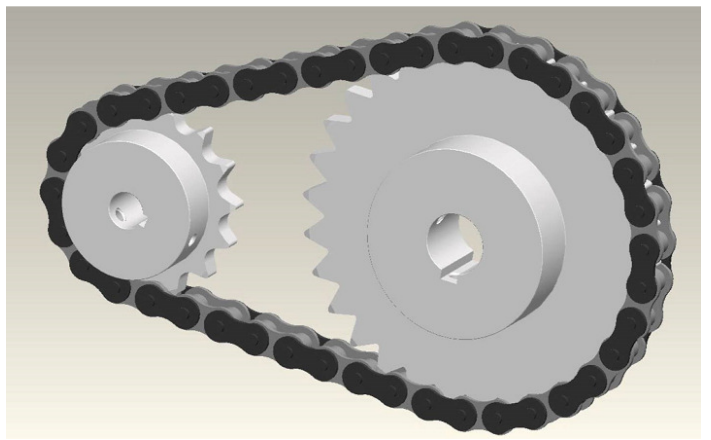

(a) Chain sprocket mechanisms

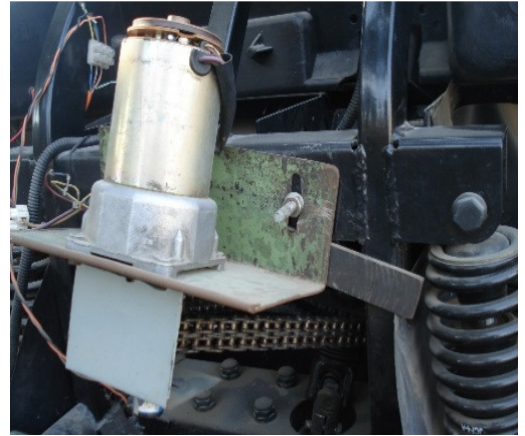

(b)Servo motor

Fig. 2. Steering control mechanisms

\section{(2) Accelerating control}

To control the car speed increasing or decreasing, a linear actuator (ball screw type)driven by $24 \mathrm{~V}$ DC motor is installed to control the accelerator pedal, cross section of the actuator, shown in Fig. 3.

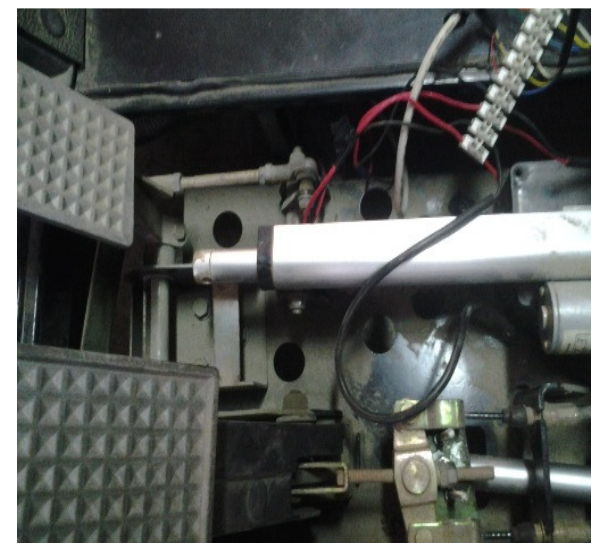

(a) linear actuator in UGV.

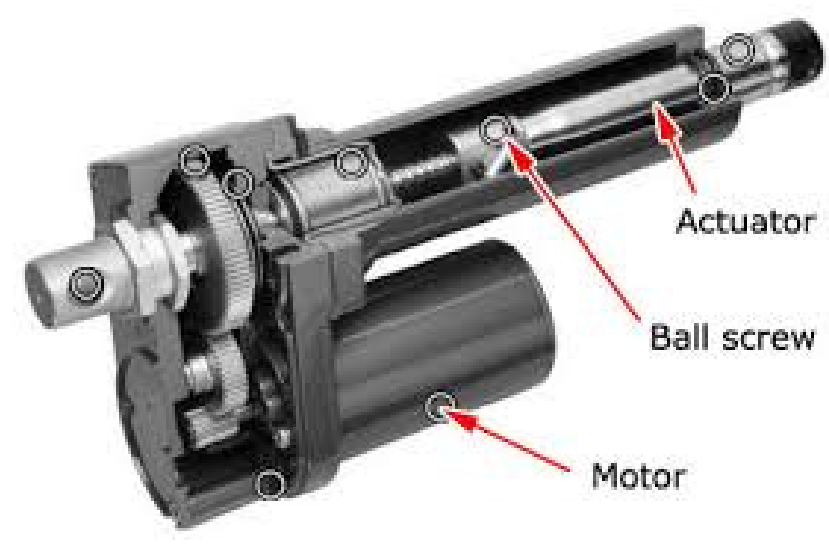

(b) Cross section of the used linear actuator (ball screw).

Fig. 3. Accelerator/ brakes control actuator.

\section{(3) Brakes control}

To control the car for complete stop, a linear actuator (ball screw type) is installed beside brake pedal, shown in Fig. 3. A calibration of the linear actuators for throttle and brakes are carried out. To enable full computer control of the vehicle actuation 
systems, the steering servo motor is controlled using PID position controller, shown in Fig. 4. Because of unknown parameters of steering servo motor the controller parameter were determined by System identification then tuned to provide the fastest, stable response at all times. The accelerator and brake control run in parallel; however, the vehicle will never attempt to accelerate while braking and vice versa.

\section{Sensors}

\section{(1) Global positioning system (GPS)}

Global Positioning System (GPS) sensor, shown in Fig. 5 is a true position sensor which will provide accurate Latitude-Longitude position. A GPS with different corrections provide position with sub-meter Circular Error Probable (CEP) accuracy. Sub-meter accuracy is sufficient for a mid-size vehicle to navigate a defined course.

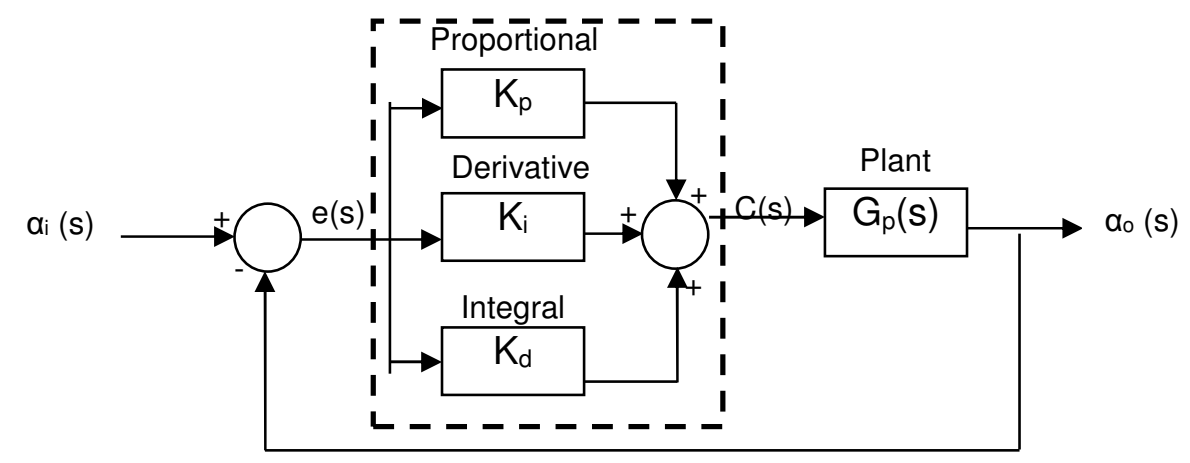

Fig. 4. PID control for motors.

\section{(2) Digital compass}

A 3DR module is used which is composed of U-Blox GPS (LEA-6H) and HMC5883L digital compass in addition to active circuitry for the ceramic patch antenna, shown in Fig. 6. There is an I2C EEPROM for configuration storage. Their Specifications are U-BloxLEA-6H module $5 \mathrm{~Hz}$ update rate, ceramic patch antenna with $25 \times 25 \times 4$ $\mathrm{mm}$, LNA and SAW filter and I2C EEPROM for configuration storage.

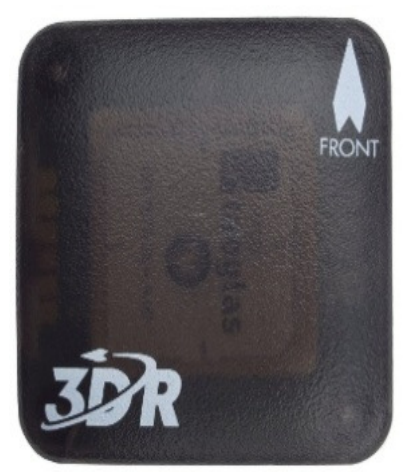

Fig. 5. Global Positioning System (GPS).

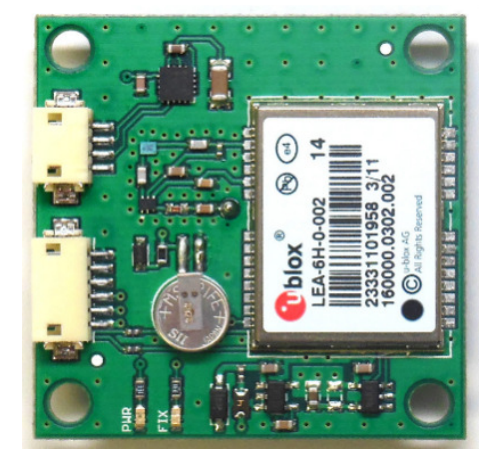

Fig. 6. HMC5883L digital compass.

\section{NAVIGATION CONTROL SYSTEM}

The navigation is the translation between two coordinates. So the navigation process 
starts by determining the required points and their Cartesian coordinates. These points are fed to the proposed navigation control system shown in Fig.7.This proposed navigation system include the longitudinal and lateral control. Longitudinal control is responsible for controlling the speed of the vehicle through the accelerator actuator. Lateral control is responsible for controlling the direction by using steering motor. The actual vehicle coordinates are obtained. The distance error longitudinal and lateral of the vehicle position is calculated according to vehicle heading. Fig. 8 presents the wheels angle error control by using PID controller with considering the mechanical limitation of the steering mechanism. Fig. 9 introduces the flow chart of wheel angle control.

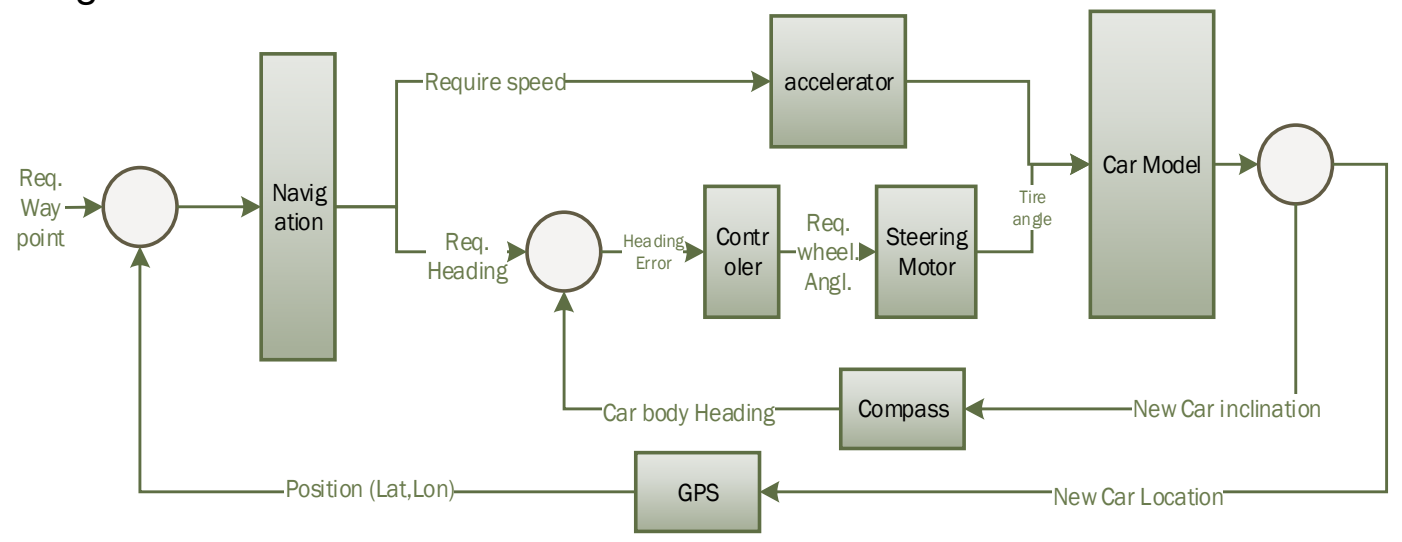

Fig. 7. Developed lateral and longitudinal navigation control.

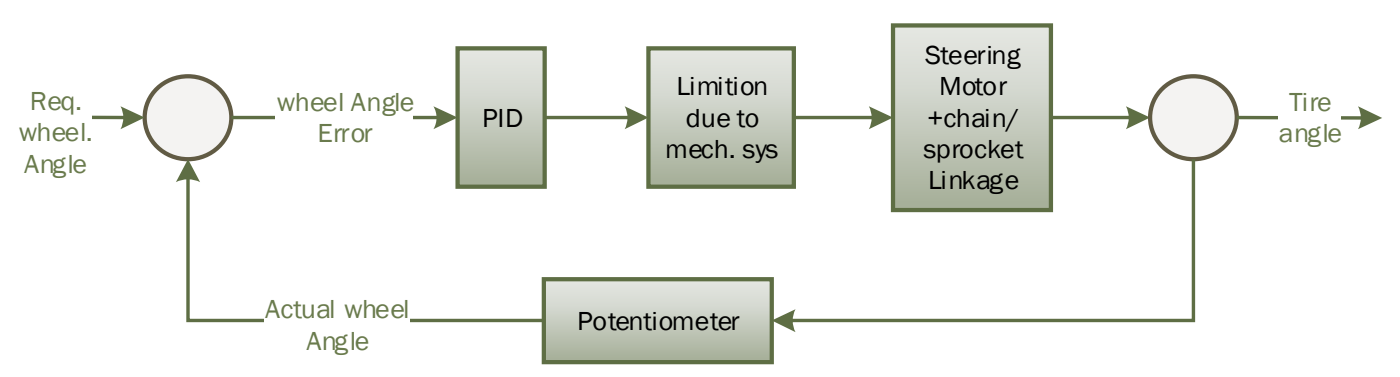

Fig. 8.Wheel angle error control.

\section{MATHEMATICAL MODEL}

The electric car is a typical Ackerman steered vehicle. It has all the wheels perpendicular to the instantaneous center of rotation due to the geometry of Ackerman steering mechanism. Therefore, the vehicle model includes four rigid wheels; the two front wheels are steerable, while the two rear wheels are fixed as shown in Fig.10. The vehicle model has two degrees of freedom to be controlled: steering angle and forward longitudinal motion. The kinematics parameters are longitudinal translation, lateral translation, yaw angle due to rotation, wheel base and wheel track $(x, y, \theta, L, d)$. The position vector of the model $r=[x, y]^{\top}$ is defined at the center of the rear wheel axle, while the angular movement of the car around its vertical axis is defined by the heading or yaw angle $(\theta)$ relative to the positive $x$-axis. The maximum allowable steering angle (left $-35<\alpha<35$ right) of the car measured 


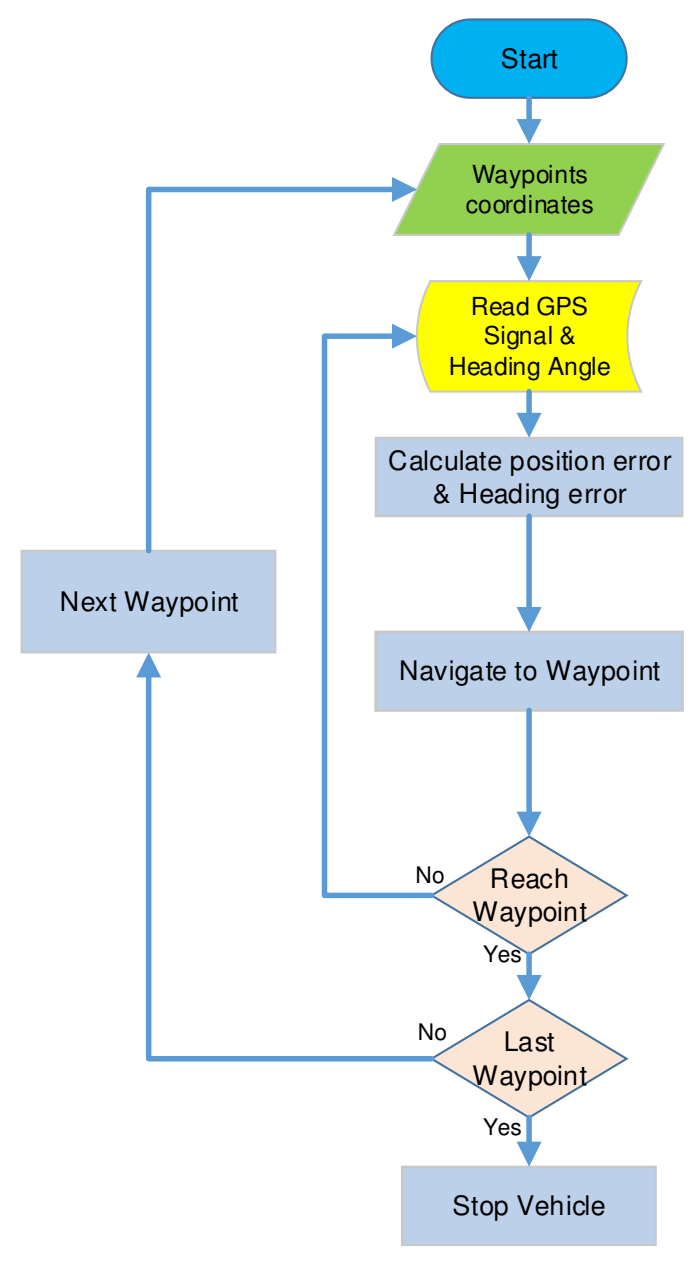

Fig. 9. Flowchart of wheel angle control.

from car heading direction. The tangential velocity of the rear axle center point is given by $(\mathrm{V})$. For the steady turning, the turning radius of the car $(R)$ is given by:

$$
\begin{gathered}
R=\frac{L}{\tan \alpha} \\
\omega=\theta^{*}=\frac{V_{s} \sin \alpha}{L} \\
x^{\prime}(t)=V_{s} \cos \alpha \cos \theta \\
y^{\prime}(t)=V_{s} \cos \alpha \sin \theta
\end{gathered}
$$

For on road automobile driving, many researchers have used the bicycle model with linearized states to design trajectory tracking controllers with consideration for the pneumatic tires [10]. The mathematical model of the vehicle is simulated as a bicycle model using MATLAB/SIMULINK, as shown in Fig. 11. 


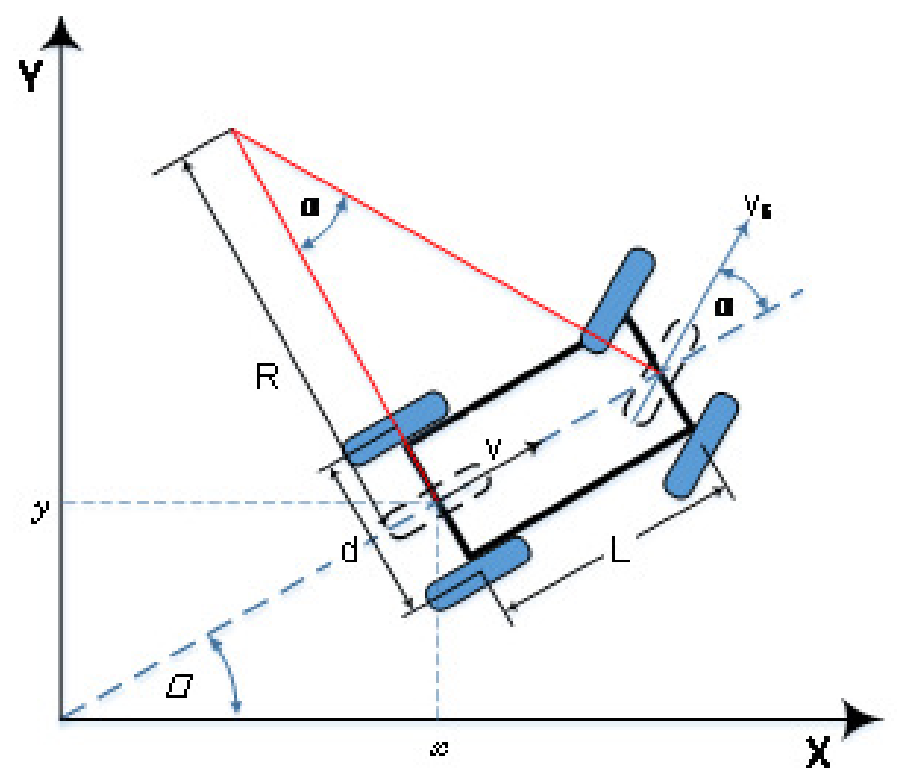

Fig. 10. Kinematic model of the car.

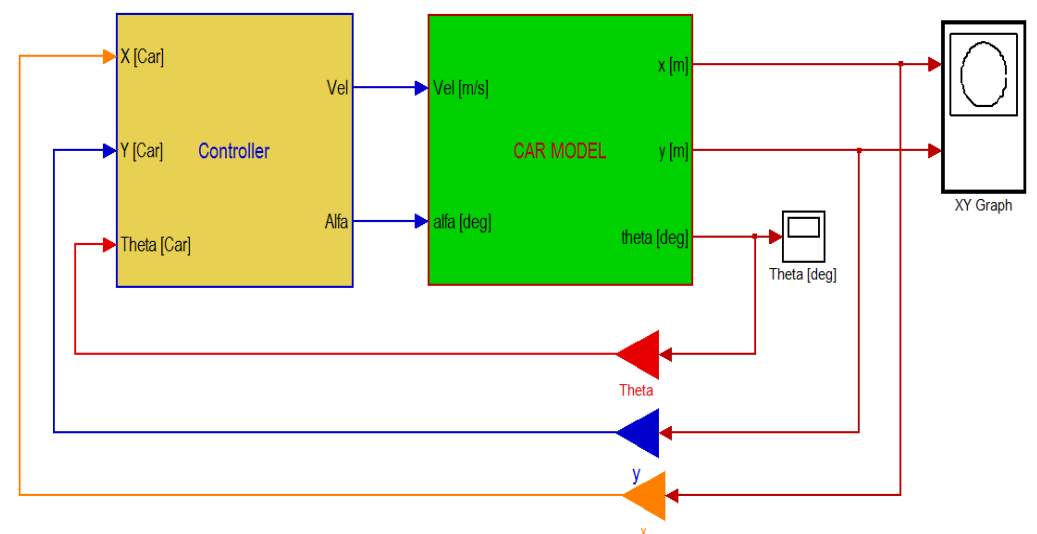

(a) MATLAB model

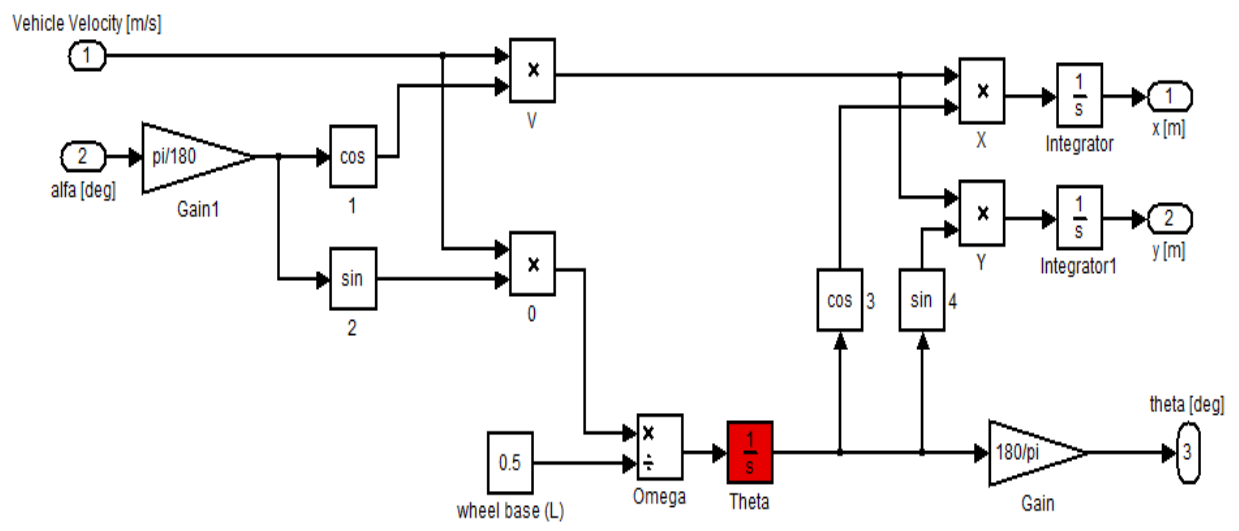

(b) SIMULINK model

Fig. 11. Bicycle model simulation. 


\section{RESULTS AND ANALYSIS}

\section{Two Points Trajectory}

Two points trajectory test is performed for examining the proposed UGV systems (actuators, sensors and navigation driving algorithm). Fig. 12 shows the actual and theoretical path between two points of navigation.

\section{Three Points Trajectory}

In this experiment, the three point's trajectory tracking task was given to the UGV to check whether the performance analysis of the proposed navigator algorithm between two points could be extended to the real system. Fig. 13(a) shows the actual coordinates of three points for navigation. The difference between simulated path, actual path and input path is shown in Fig. 13 (b). The results show that the simulated path is coincident over the input path. There is a little difference between actual path and required path. This is due to linearization of the bicycle model and neglecting the effect of tire model.

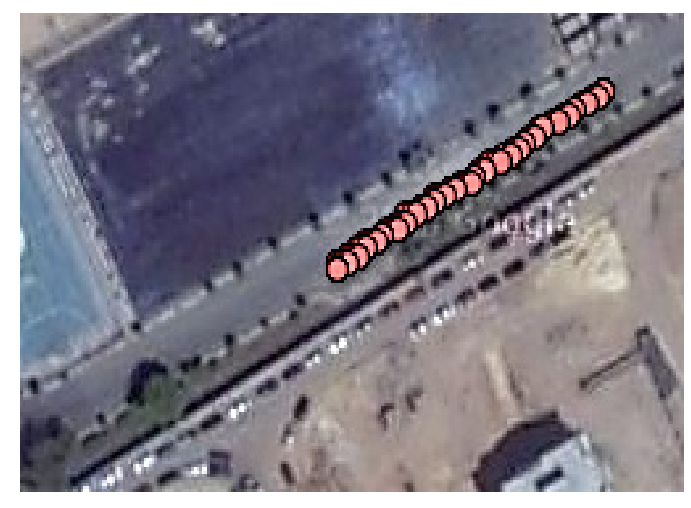

(a) Actual coordinates

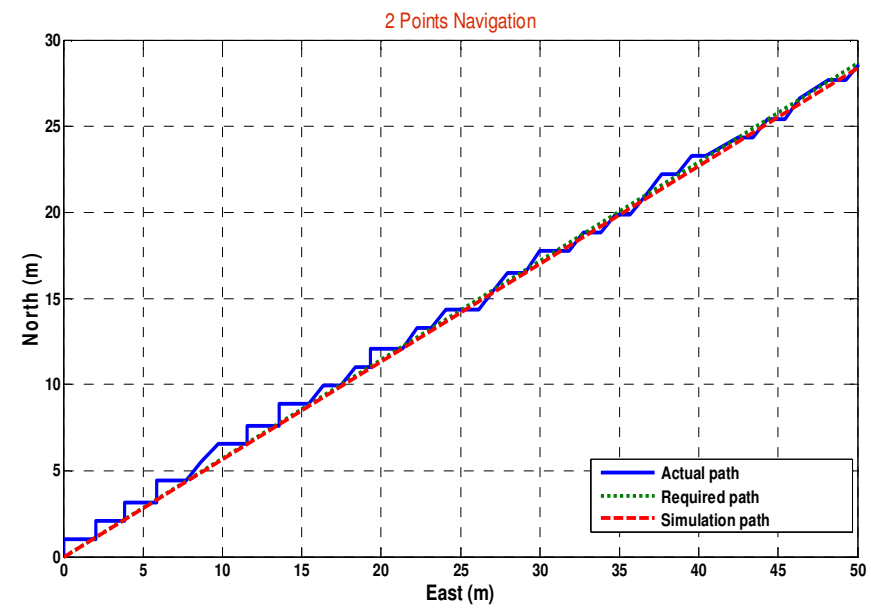

(b) Actual, required and simulated path

Fig. 12. Actual and theoretical path navigation between two points.

\section{Four Points Trajectory}

The performance of the proposed tracking method was valued through the simulation scenario with the desired four point's trajectory to follow, as shown in Fig. 14 (a). The difference between simulated path, actual path and input path is shown in Fig. 14 (b). As the UGV dynamics and its input constraints, it was difficult for the UGV to track the given trajectory perfectly because of the sharp $90^{\circ}$ turns at the corners. 


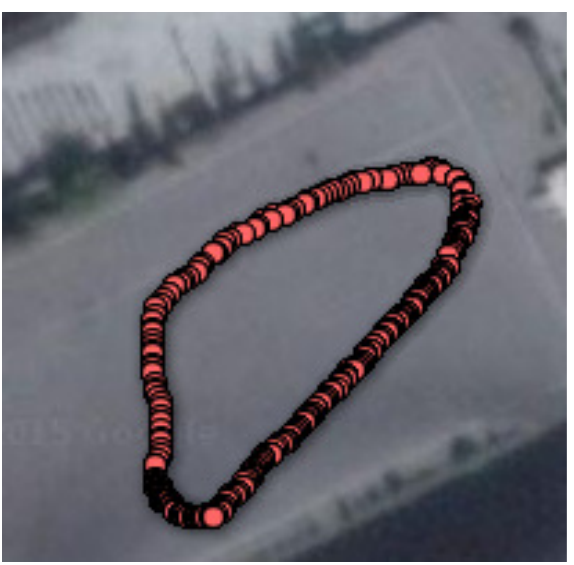

(a) Actual coordinates

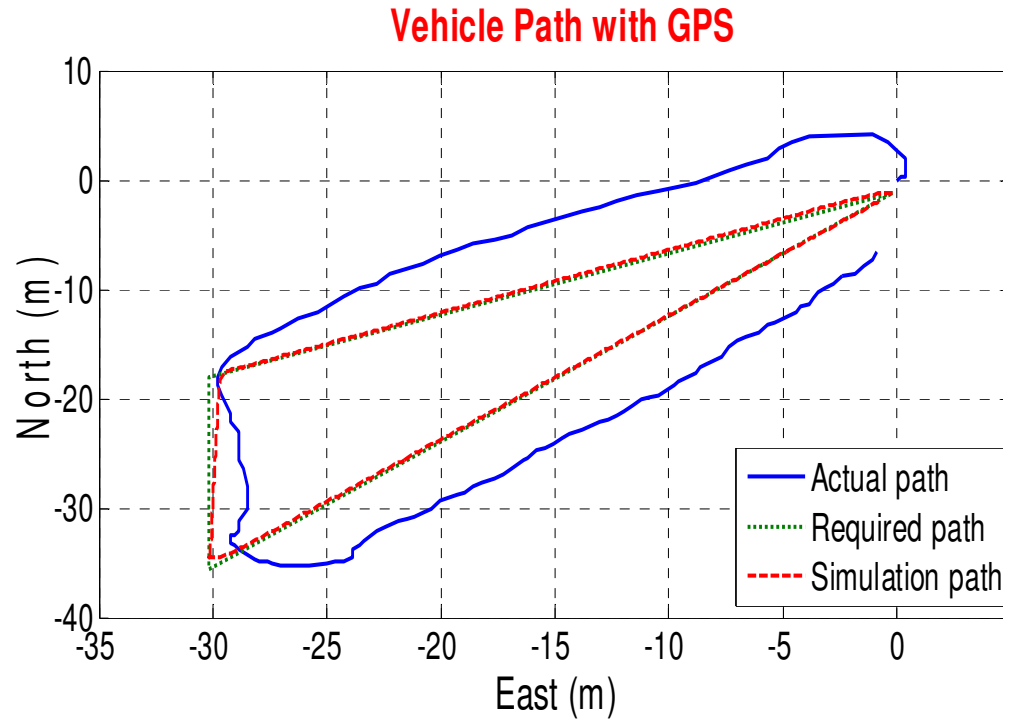

(b) Actual, required and simulated path

Fig.13.Actual and theoretical path navigation between three points.

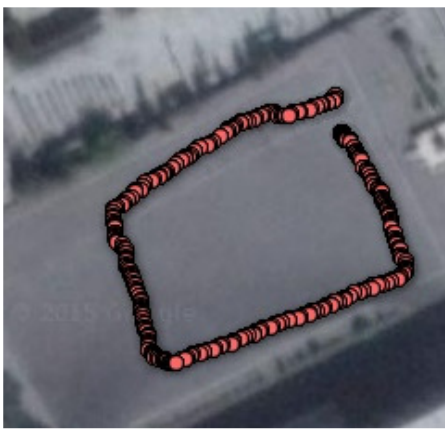

(a) Actual coordinates.

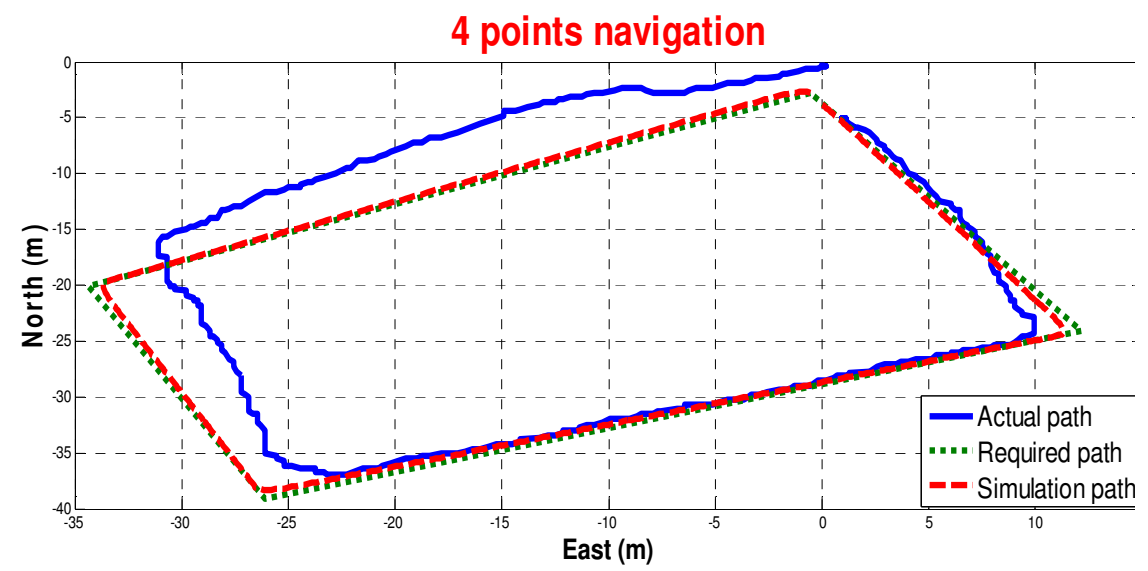

(b) Actual, requiredand simulated path

Fig.14.Actual and theoretical path navigation between four points.

\section{CONCLUSIONS}

An electric car is converted to be unmanned vehicle by using three actuators for steering, accelerating and braking systems. A position controller was implemented to prevent simultaneous operation of accelerator and brake actuators.

A mathematical model for Ackerman steering is modeled and simulated using MATLAB/SIMULINK. The proposed navigation system is implemented based on GPS integrated with a digital compass. The navigation system includes a longitudinal control for car speed and a lateral control for direction. From simulation results, the simulation path is coincident on required path. Form experimental testing results there are good correlations for actual and required navigation between two points, 
three points and four points. Commercial sensors and low cost actuators in addition to linearization of the bicycle model are the reasons of that little difference between actual vehicle path and required path.

\section{REFERENCES}

[1] J. R. Strachan, "NOVA: Nottingham Off-road Vehicle Architecture," University of Nottingham, 2009.

[2] X. Liu and B. Dai, "The latest status and development trends of military unmanned ground vehicles," in Chinese Automation Congress (CAC), 2013, 2013, pp. 533-537.

[3] H. Lim, Y. Kang, C. Kim, and J. Kim, "Experimental verification of nonlinear model predictive tracking control for six-wheeled unmanned ground vehicles," International Journal of Precision Engineering and Manufacturing, vol. 15, pp. 831-840, 2014/05/01 2014.

[4] M. Z. H. Noor, S. A. S. M. Zain, and L. Mazalan, "Design and development of remote-operated multi-direction Unmanned Ground Vehicle (UGV)," in System Engineering and Technology (ICSET), 2013 IEEE 3rd International Conference on, 2013, pp. 188-192.

[5] T. Czapla and J. Wrona, "Technology Development of Military Applications of Unmanned Ground Vehicles," in Vision Based Systemsfor UAV Applications. vol. 481, A. Nawrat and Z. Kuś, Eds., ed: Springer International Publishing, 2013, pp. 293-309.

[6] G. Wilson, A. Ramirez-Serrano, M. Mustafa, and K. Davies, "Velocity Selection for High-Speed UGVs in Rough Unknown Terrains Using Force Prediction," in Intelligent Robotics and Applications. vol. 7507, C.-Y. Su, S. Rakheja, and H. Liu, Eds., ed: Springer Berlin Heidelberg, 2012, pp. 387-396.

[7] J. P. Lee, "Future Unmanned System Design for Reliable Military Operations," International Journal of Control \& Automation, vol. 5, 2012.

[8] A. Lacaze, K. Murphy, M. Del Giorno, and K. Corley, "Reconnaissance and Autonomy for Small Robots (RASR) team: MAGIC 2010 challenge," Journal of Field Robotics, vol. 29, pp. 729-744, 2012.

[9] M. Young-Geun, K. Moon-Sik, and L. Min-Cheol, "Development of speed control and path following algorithm based on RTK-DGPS," 2011 11th International Conference on Control, Automation and Systems, pp. 734-737, 2011.

[10] Gillespie, T. D., Fundamentals of Vehicle Dynamics, Society of Automotive Engineers, Warrendale, PA, 1992. 